\title{
Boundedness of Sublinear Operators with Rough Kernels on Weighted Morrey Spaces
}

\author{
Shaoguang Shi and Zunwei Fu \\ Department of Mathematics, Linyi University, Linyi 276005, China \\ Correspondence should be addressed to Zunwei Fu; zwfu@mail.bnu.edu.cn \\ Received 18 January 2013; Accepted 11 March 2013 \\ Academic Editor: Dashan Fan
}

Copyright (C) 2013 S. Shi and Z. Fu. This is an open access article distributed under the Creative Commons Attribution License, which permits unrestricted use, distribution, and reproduction in any medium, provided the original work is properly cited.

The aim of this paper is to get the boundedness of a class of sublinear operators with rough kernels on weighted Morrey spaces under generic size conditions, which are satisfied by most of the operators in classical harmonic analysis. Applications to the corresponding commutators formed by certain operators and BMO functions are also obtained.

\section{Introduction and Main Results}

Given a function $\Omega$ over the unit sphere $S^{n-1}$ of $\mathbb{R}^{n}(n \geq 2)$ equipped with the normalized Lebesgue measure $d \sigma$ and $x^{\prime}=$ $x /|x|$, a Calderón-Zygmund singular integral operator with rough kernel was given by

$$
T_{\Omega} f(x)=\text { p.v. } \int_{\mathbb{R}^{n}} \frac{\Omega(x-y)}{|x-y|^{n}} f(y) d y
$$

and a related maximal operator

$$
M_{\Omega} f(x)=\sup _{r>0} \frac{1}{r_{n}} \int_{B(x, r)} \Omega(x-y) f(y) d y,
$$

where $\Omega$ is homogeneous of degree zero and satisfies

$$
\begin{gathered}
\Omega \in L^{r}\left(S^{n-1}\right), \quad 1<r \leq \infty \\
\int_{S^{n-1}} \Omega\left(x^{\prime}\right) d x^{\prime}=0 .
\end{gathered}
$$

When $\Omega$ is a smooth kernel and $T_{\Omega}$ a standard CalderónZygmund singular integral operator which has been fully studied by many papers, a classical survey work; see, for example, [1].

For simplicity of notation, $\Omega$ is always homogeneous of degree zero and satisfies (3) and (4) throughout this paper if there are no special instructions. Here and in what follows, for $x_{0} \in \mathbb{R}^{n}, r>0$, and $\lambda>0, B=B\left(x_{0}, r\right)$ denotes the ball centered at $x_{0}$ with radius $r$ and $\lambda B=$ $B\left(x_{0}, \lambda r\right)$. When $\Omega$ satisfies some size conditions, the kernel of the operator $T_{\Omega}$ has no regularity, and so the operator $T_{\Omega}$ is called rough singular integral operator. In recent years, a variety of operators related to the singular integrals for Calderón-Zygmund, but lacking the smoothness required in the classical theory, have been studied. Duoandikoetxea [2] studied the norm inequalities for $T_{\Omega}$ in homogeneous case on weighted $L^{p}(1<p<\infty)$ spaces. For more corresponding works, we refer the reader to [3-8] and the references therein.

In [9], $\mathrm{Hu}$ et al. considered some more general sublinear operators with rough kernels which satisfy

$$
\begin{aligned}
& \left|\mathscr{T}_{\Omega} f(x)\right| \\
& \quad \leq C \int_{\mathbb{R}^{n}} \frac{|\Omega(x-y) f(y)|}{|x-y|^{n}} d y, \quad x \notin \operatorname{supp} f
\end{aligned}
$$

for $f \in L^{1}\left(\mathbb{R}^{n}\right)$ with compact support. Condition (5) was first introduced by Soria and Weiss [10]. Inequality (5) is satisfied by many operators with rough kernels in classical harmonic analysis, such as $T_{\Omega}$ (see [11]) and the oscillatory singular integral operator

$$
\begin{aligned}
& \bar{T}_{\Omega} f(x) \\
& \quad=\text { p.v. } \int_{\mathbb{R}^{n}} e^{i P(x, y)} \frac{\Omega(x-y)}{|x-y|^{n}} f(y) d y, \quad x \notin \operatorname{supp} f,
\end{aligned}
$$


where the phase is a polynomial. The boundedness of $\bar{T}_{\Omega}$ on weighted $L^{p}\left(\mathbb{R}^{n}\right)(1 \leq p<\infty)$ spaces was fully studied by Ojanen in his doctoral dissertation [12].

Let $D_{k}=\left\{x \in \mathbb{R}^{n}:|x| \leq 2^{k}\right\}$ and let $A_{k}=D_{k} \backslash D_{k-1}$ for $k \in Z$. Throughout this paper, we will denote by $\chi_{E}$ the characteristic function of the set $E$. Inspired by the works of $[6,13]$, in this paper, we consider some sublinear operators under some size conditions (the following (7) and (8)) which are more general than (5):

$$
\left|\mathscr{T}_{\Omega} f(x)\right| \leq C|x|^{-n} \int_{\mathbb{R}^{n}}|\Omega(x-y) f(y)| d y,
$$

when supp $f \subseteq A_{k}$ and $|x| \geq 2^{k+1}$ with $k \in \mathbb{Z}$ and

$$
\left|\mathscr{T}_{\Omega} f(x)\right| \leq C 2^{-k n} \int_{\mathbb{R}^{n}}|\Omega(x-y) f(y)| d y,
$$

when supp $f \subseteq A_{k}$ and $|x| \leq 2^{k-1}$ with $k \in \mathbb{Z}$, respectively. It is worth pointing out that $M_{\Omega}$ satisfies conditions (7) and (8). Also, condition (5) implies the size conditions (7) and (8) since $|x-y|>|x| / 2$ when $|x| \geq 2^{k+1}$ and supp $f \subseteq A_{k}$ while supp $f \subseteq A_{k}$ and $|x| \leq 2^{k-1}$ imply $|x-y|>|y| / 2$.

The topic of this paper is intended as an attempt to study the boundedness of sublinear operators with rough kernels which satisfy (7) and (8) on weighted Morrey spaces. We first recall some definitions and notations for weighted spaces. The Muckenhoupt classes $A_{p}$ and $A_{(p, q)}[14]$ contain the functions $w$ which satisfy

$$
\begin{aligned}
& A_{p}: \sup _{B}\left(\frac{1}{|B|} \int_{B} w(x) d x\right) \\
& \quad \times\left(\frac{1}{|B|} \int_{B} w(x)^{1-p^{\prime}} d x\right)^{p-1} \leq C, \quad 1<p<\infty, \\
& A_{(p, q)}: \sup _{B}\left(\frac{1}{|B|} \int_{B} w(x)^{q} d x\right)^{1 / q} \\
& \quad \times\left(\frac{1}{|B|} \int_{B} w(x)^{-p^{\prime}} d x\right)^{1 / p^{\prime}} \leq C, \quad 1<p, q<\infty,
\end{aligned}
$$

respectively, where $1 / p+1 / p^{\prime}=1$. For $p=1$, the $A_{1}$ and $A_{(1, q)}(1<q<\infty)$ weights are defined by

$$
\begin{gathered}
M w(x) \leq C w(x), \\
A_{(1, q)}: \sup _{B}\left(\frac{1}{|B|} \int_{B} w(x)^{q} d x\right)^{1 / q} \\
\times\left(\operatorname{ess} \sup _{B} \frac{1}{w(x)}\right) \leq C,
\end{gathered}
$$

respectively. Here ess sup and the following essinf are the abbreviations of essential supremum and essential infimum, respectively. Clearly, $w \in A_{1}$ if and only if there is a constant $C>0$ such that

$$
\frac{1}{|B|} \int_{B} w(x) d x \leq \text { Cessinf } \sin _{B} w(x) .
$$

In [15], Komori and Shirai introduced a weighted Morrey space, which is a natural generalization of weighted Lebesgue space, and investigated the boundedness of classical operators in harmonic analysis. Let $1 \leq p<\infty, 0<\lambda<1$ and let $w$ be a weight function. Then the weighted Morrey space $M_{p, \lambda}(w)$ is defined by

$$
\begin{aligned}
M_{p, \lambda}(w)= & \left\{f:\|f\|_{M_{p, \lambda}(w)}\right. \\
& \left.=\sup _{B}\left(\frac{1}{w(B)^{\lambda}} \int_{B}|f(x)|^{p} w(x) d x\right)^{1 / p}<\infty\right\},
\end{aligned}
$$

where $w(B)=\int_{B} w(x) d x$. For $w \in A_{p}(1 \leq p<\infty)$, if $\lambda=0$, then $M_{p, 0}(w)=L^{p}(w)$ while $\lambda=1$ implies $M_{p, 1}(w)=$ $L^{\infty}(w)$.

Now, we formulate our major results of this paper as follows.

Theorem 1. Let $0<\lambda<1,1<r \leq \infty$, and $r^{\prime} \leq p<\infty$ and let a sublinear operator $\mathscr{T}_{\Omega}$ satisfy (7) and (8). If $\mathscr{T}_{\Omega}$ is bounded on $L^{p}(w)$ with $w \in A_{p / r}$, then $\mathscr{T}_{\Omega}$ is bounded on $M_{p, \lambda}(w)$.

When $p=1$, we have the following theorem.

Theorem 2. Let $1<r<\infty$ and $1 / r+\lambda<1$ and let $\mathscr{T}_{\Omega}$ satisfy (7) and (8). Then if $\mathscr{T}_{\Omega}$ is bounded from $L^{1}(w)$ to $L^{1, \infty}(w)$ with $w \in A_{1}$, there exists a constant $C>0$ such that for all $\mu>0$ and all balls $B$,

$$
\begin{aligned}
w(\{x & \left.\left.\in B: \mathscr{T}_{\Omega} f(x)>\mu\right\}\right) \\
& \leq C \mu^{-1}\|f\|_{M_{1, \lambda}(w)} w(B)^{\lambda} .
\end{aligned}
$$

In the fractional case, we need to consider a weighted Morrey space with two weights which is also introduced by Komori and Shirai in [15]. Let $1 \leq p<\infty, 0<\lambda<1$. For two weights $w_{1}$ and $w_{2}$,

$$
\begin{aligned}
M_{p, \lambda}\left(w_{1}, w_{2}\right)=\{f & :\|f\|_{M_{p, k}\left(w_{1}, w_{2}\right)} \\
& =\sup _{B}\left(\frac{1}{w_{2}(B)^{\lambda}} \int_{B}|f(x)|^{p} w_{1}(x) d x\right)^{1 / p} \\
& <\infty\} .
\end{aligned}
$$

If $w_{1}=w_{2}=w$, we write $M_{p, \lambda}\left(w_{1}, w_{1}\right)=M_{p, \lambda}\left(w_{2}, w_{2}\right)=$ $M_{p, \lambda}(w)$.

We can get similar results for fractional integrals following the line of Theorems 1 and 2. 
Theorem 3. Let $0<\alpha<n, 1 \leq r<\infty$, and $0<\lambda<$ 1. Suppose that a sublinear operator $\mathscr{T}_{\alpha, \Omega}$ satisfies the size conditions

$$
\begin{aligned}
& \left|\mathscr{T}_{\alpha, \Omega} f(x)\right| \\
& \quad \leq C|x|^{-(n-\alpha)} \int_{\mathbb{R}^{n}}|\Omega(x-y) f(y)| d y
\end{aligned}
$$

when supp $f \subseteq A_{k}$ and $|x| \geq 2^{k+1}$ with $k \in Z$ and

$$
\begin{aligned}
& \quad\left|\mathscr{T}_{\alpha, \Omega} f(x)\right| \\
& \quad \leq C 2^{-k(n-\alpha)} \int_{\mathbb{R}^{n}}|\Omega(x-y) f(y)| d y
\end{aligned}
$$

when supp $f \subseteq A_{k}$ and $|x| \leq 2^{k-1}$ with $k \in Z$. Then one has the following.

(a) If $\mathscr{T}_{\alpha, \Omega}$ maps $L^{p}\left(w^{p}\right)$ into $L^{q}\left(w^{q}\right)$ with $w \in$ $A_{\left(p / r^{\prime}, q\right)}$, then $\mathscr{T}_{\alpha, \Omega}$ is bounded from $M_{p, \lambda}\left(w^{p}, w^{q}\right)$ to $M_{q, q \lambda / p}\left(w^{q}\right)$, where $r^{\prime} \leq p<n / \alpha, 1 / q=1 / p-\alpha / n$ and $p \leq q \leq \infty$.

(b) If $\mathscr{T}_{\alpha, \Omega}$ is bounded from $L^{1}(w)$ to $L^{q, \infty}\left(w^{q}\right)$ with $w \in$ $A_{(1, q)}$ and $1 / r+\lambda<1$, then there exists a constant $C>0$ such that for all $\mu>0$ and all balls $B$,

$$
\begin{aligned}
w(\{x & \left.\left.\in B: \mathscr{T}_{\alpha, \Omega} f(x)>\mu\right\}\right)^{1 / q} \\
& \leq C \mu^{-1}\|f\|_{M_{1, \lambda}\left(w, w^{q}\right)} w(B)^{\lambda}
\end{aligned}
$$

where $1<q<\infty$.

We emphasize that (15) and (16) are weaker conditions than the following condition:

$$
\begin{aligned}
& \left|\mathscr{T}_{\alpha, \Omega} f(x)\right| \\
& \quad \leq C \int_{\mathbb{R}^{n}} \frac{|\Omega(x-y) f(y)|}{|x-y|^{n-\alpha}} d y, \quad 0<\alpha<n
\end{aligned}
$$

for any integral function $f$ with compact support. Condition (18) is satisfied by most fractional integral operators with rough kernels, such as the fractional integral operators of Muckenhoupt and Wheeden [16]:

$$
\begin{aligned}
& \mathscr{T}_{\alpha, \Omega} f(x) \\
& \quad=\int_{\mathbb{R}^{n}} \frac{\Omega(x-y) f(y)}{|x-y|^{n-\alpha}} d y, \quad 0<\alpha<n .
\end{aligned}
$$

For some mapping properties of $\mathscr{T}_{\alpha, \Omega}$ on various kinds of function spaces, see [17-19] and the references therein.

We end this section with the outline of this paper. Section 2 contains the proofs of Theorems 1 and 3; this part is partly motivated by the methods in [20] dealing with the case of the Lebesgue measure. In Section 3, we extend the corresponding results to commutators of certain sublinear operators.

\section{Boundedness of Sublinear Operators}

Proofs of Theorems 1 and 3 depend heavily on some properties of $A_{p}$ weights, which can be found in any papers or any books dealing with weighted boundedness for operators in harmonic analysis, such as [1]. For the convenience of the reader we collect some relevant properties of $A_{p}$ weights without proofs, thus making our exposition self-contained.

Lemma 4. Let $1 \leq p<\infty$ and $w \in A_{p}$. Then the following statements are true.

(a) There exists a constant $C$ such that

$$
w(2 B) \leq C w(B)
$$

where $w$ satisfies this condition; one says $w$ satisfies the doubling condition.

(b) There exists a constant $C>1$ such that

$$
w(2 B) \geq C w(B),
$$

where $w$ satisfies this condition; one says $w$ satisfies the reverse doubling condition.

(c) There exist two constants $C$ and $r>1$ such that the following reverse Hölder inequality holds for every ball $B \subset \mathbb{R}^{n}$ :

$$
\left(\frac{1}{|B|} \int_{B} w(x)^{r} d x\right)^{1 / r} \leq C\left(\frac{1}{|B|} \int_{B} w(x) d x\right) .
$$

(d) For all $\lambda>1$, one has

$$
w(\lambda B) \leq C \lambda^{n p} w(B) .
$$

(e) There exist two constants $C$ and $\delta>0$ such that for any measurable set $Q \subset B$

$$
\frac{w(Q)}{w(B)} \leq C\left(\frac{|Q|}{|B|}\right)^{\delta}
$$

if $w$ satisfies (24); one says $w \in A_{\infty}$.

(f) For all $p<q<\infty$, one has

$$
A_{\infty}=\cup_{p} A_{p}, \quad A_{p} \subset A_{q} .
$$

The following lemma about the rough kernel $\Omega$ is essential to our proofs. One can find its proof in [21].

Lemma 5. Let $\Omega \in L^{r}\left(S^{n-1}\right)$ with $1 \leq r<\infty$. Then the following statements are true.

(a) If $x \in A_{k}$ and $j \geq k+1$, then $\int_{A_{j}}|\Omega(x-y)|^{r} d y \leq C 2^{j n}$.

(b) If $y \in A_{k}$ and $k \geq j+1$, then $\int_{A_{j}}|\Omega(x-y)|^{r} d x \leq$ $C 2^{k(n-1)+j}$. 
Proof of Theorem 1. Let $1<r^{\prime} \leq p<\infty, w \in A_{p / r^{\prime}}$, and $0<\lambda<1$. Our task is to show

$$
\frac{1}{w(B)^{\lambda}} \int_{B}\left|\mathscr{T}_{\Omega} f(x)\right|^{p} w(x) d x \leq C\|f\|_{M_{p, \lambda}(w)}^{p}
$$

For a fixed ball $B=B\left(x_{0}, r\right)$, there is no loss of generality in assuming $r=1$. We decompose $f=f \chi_{2 B}+f \chi_{(2 B)^{c}}:=f_{1}+f_{2}$. Since $\mathscr{T}_{\Omega}$ is a sublinear operator, so we get

$$
\begin{aligned}
& \frac{1}{w(B)^{\lambda}} \int_{B}\left|\mathscr{T}_{\Omega} f(x)\right|^{p} w(x) d x \\
& \leq \frac{C}{w(B)^{\lambda}} \int_{B}\left|\mathscr{T}_{\Omega} f_{1}(x)\right|^{p} w(x) d x \\
& \quad+\frac{C}{w(B)^{\lambda}} \int_{B}\left|\mathscr{T}_{\Omega} f_{2}(x)\right|^{p} w(x) d x \\
& :=I+I I .
\end{aligned}
$$

By the assumption on $\mathscr{T}_{\Omega}$ and (25), we can obtain

$$
\begin{aligned}
I & \leq \frac{C}{w(B)^{\lambda}} \int_{\mathbb{R}^{n}}\left|\mathscr{T}_{\Omega} f_{1}(x)\right|^{p} w(x) d x \\
& \leq \frac{C}{w(B)^{\lambda}} \int_{2 B}|f(x)|^{p} w(x) d x \\
& \leq C\|f\|_{M_{p, \lambda}(w)}^{p}
\end{aligned}
$$

For the term $I I$, by (8) we have

$$
I I \leq \frac{C}{w(B)^{\lambda}} \int_{B}\left|\sum_{k=1}^{\infty} 2^{-k n} \mathscr{T}_{\Omega, k} f(x)\right|^{p} w(x) d x,
$$

where

$$
\mathscr{T}_{\Omega, k} f(x)=\int_{A^{k+1}}|\Omega(x-y) f(y)| d y .
$$

We distinguish two cases according to the size of $p$ and $r$ to get the estimates for $\mathscr{T}_{\Omega, k}$.

Case $1\left(p>r^{\prime}\right)$. In this case, $w \in A_{p / r^{\prime}}$ implies that

$$
\int_{B} w^{-r^{\prime} /\left(p-r^{\prime}\right)} d y \leq \frac{|B|^{p /\left(p-r^{\prime}\right)}}{w(B)^{r^{\prime} /\left(p-r^{\prime}\right)}} .
$$

By (31), Hölder's inequality, and Lemma 5, we have

$$
\begin{aligned}
\mathscr{T}_{\Omega, k} f(x) \leq & C\left(\int_{A^{k+1}}|\Omega(x-y)|^{r} d y\right)^{1 / r} \\
& \times\left(\int_{2^{k+1} B}|f(y)|^{r^{\prime}} d y\right)^{1 / r^{\prime}} \\
\leq & C 2^{(k+1) n / r} \\
& \times\left(\int_{2^{k+1} B}|f(y)|^{r^{\prime}} w(y)^{r^{\prime} / p} w(y)^{-r^{\prime} / p} d y\right)^{1 / r^{\prime}} \\
\leq & C 2^{(k+1) n / r}\left(\int_{2^{k+1} B}^{\left.|f(y)|^{p} w(y) d y\right)^{1 / p}}\right. \\
& \times\left(\int_{2^{k+1} B} w(y)^{-r^{\prime} /\left(p-r^{\prime}\right)} d y\right)^{\left(p-r^{\prime}\right) /\left(p r^{\prime}\right)} \\
\leq & C\|f\|_{M_{p, \lambda}(w)} 2^{(k+1) n / r} \frac{\left|2^{k+1} B\right|^{1 / r^{\prime}}}{w\left(2^{k+1} B\right)^{(1-\lambda) / p}} \\
\leq & C\|f\|_{M_{p, \lambda}(w)} \frac{2^{(k+1) n}}{w\left(2^{k+1} B\right)^{(1-\lambda) / p}} .
\end{aligned}
$$

Case $2\left(p=r^{\prime}\right)$. In this case, $w \in A_{1}$ implies that

$$
\left(\text { ess } \inf _{x \in 2^{k+1} B} w(x)\right)^{-1} \leq \frac{\left|2^{k+1} B\right|}{w\left(2^{k+1} B\right)},
$$

which in combination with the Hölder inequality and Lemma 5 yields that

$$
\begin{aligned}
\mathscr{T}_{\Omega, k} f(x) \leq & C 2^{(k+1) n / r} \\
& \times\left(\int_{2^{k+1} B}|f(y)|^{p} w(y) w(y)^{-1} d y\right)^{1 / p} \\
\leq & C\|f\|_{M_{p, \lambda}(w)} \frac{2^{(k+1) n}}{w\left(2^{k+1} B\right)^{(1-\lambda) / p}} .
\end{aligned}
$$

Substituting (32) and (34) into (29), we can assert that

$$
\begin{aligned}
I I & \leq C\|f\|_{M_{p, \lambda}(w)}^{p}\left(\sum_{k=1}^{\infty} \frac{w(B)^{(1-\lambda) / p}}{w\left(2^{k+1} B\right)^{(1-\lambda) / p}}\right)^{p} \\
& \leq C\|f\|_{M_{p, \lambda}(w)}^{p}
\end{aligned}
$$

where we have used (21) in the last inequality. Combining (28) and (29), we obtain the proof of Theorem 1.

Proof of Theorem 2. The task is now to show the following inequality:

$$
\begin{aligned}
& \sup _{\mu>0} \frac{\mu}{w(B)^{\lambda}} w\left(\left\{x \in B:\left|\mathscr{T}_{\Omega} f(x)\right|>\mu\right\}\right) \\
& \leq C\|f\|_{M_{p, \lambda}(w)}^{p} .
\end{aligned}
$$


In order to get this inequality, it will be necessary to decompose $f=f \chi_{2 B}+f \chi_{(2 B)^{c}}:=f_{1}+f_{2}$ with $B$ as in Theorem 1 . Since $\mathscr{T}_{\Omega}$ is a sublinear operator, we can rewrite

$$
\begin{aligned}
& w\left(\left\{x \in B:\left|\mathscr{T}_{\Omega} f(x)\right|>\mu\right\}\right) \\
& \leq w\left(\left\{x \in B:\left|\mathscr{T}_{\Omega} f_{1}(x)\right|>\frac{\mu}{2}\right\}\right) \\
& \quad+w\left(\left\{x \in B:\left|\mathscr{T}_{\Omega} f_{2}(x)\right|>\frac{\mu}{2}\right\}\right) \\
& :=J+J J .
\end{aligned}
$$

An application of (20) and the weighted weak type estimates for $\mathscr{T}_{\Omega}$ yield that

$$
J \leq C \mu^{-1}\|f\|_{M_{1, \lambda}}(w) w(B)^{\mu} .
$$

To estimate the term $J J$, we note that

$$
\begin{aligned}
J J \leq \frac{C}{\mu} & \int_{\left\{x \in B:\left|\mathcal{T}_{\Omega} f(x)\right|>\mu / 2\right\}} \\
& \times\left|\sum_{k=1}^{\infty} 2^{-k n} \mathscr{T}_{\Omega, k} f(x)\right| w(x) d x .
\end{aligned}
$$

By (22), (33), and the Hölder inequality, we can estimate $J J$ as

$$
\begin{aligned}
J \leq & \frac{C}{\mu} \sum_{k=1}^{\infty} 2^{-k n} \\
& \times \int_{2^{k+1} B} \int_{B}|\Omega(x-y)| w(x) d x|f(y)| d y \\
\leq & \frac{C w(B)}{\mu} \sum_{k=1}^{\infty} 2^{-k n+k(n-1) / r} \\
& \times \int_{2^{k+1} B}|f(y)| w(y) w(y)^{-1} d y \\
\leq & \frac{C w(B)}{\mu}\|f\|_{M_{1, \lambda}}(w) \\
& \times \sum_{k=1}^{\infty} w\left(2^{k+1} B\right)^{\lambda}(\text { ess inf } w)^{-1} \\
\leq & \frac{C}{\mu}\|f\|_{M_{1, \lambda}}(w) \\
\leq & \frac{C}{\mu}\|f\|_{M_{1, \lambda}}(w) w(B)^{\lambda} . \\
& \times \sum_{k=1}^{\infty} 2^{k n / r-k n(1-\lambda)} w(B)^{\lambda} \\
&
\end{aligned}
$$

Combining these inequalities for $J$ and $J J$, we have completed the proof of Theorem 2 .

Proof of Theorem 3. We can use the similar arguments as in the proof of Theorem 1 and Theorem 2. For the proof of $(a)$, it suffices to show that

$$
\frac{1}{w^{q}(B)^{q \lambda / p}} \int_{B}\left|\mathscr{T}_{\alpha, \Omega} f(x)\right|^{q} w(x)^{q} d x \leq C\|f\|_{M_{p, \lambda}\left(w^{p}, w^{q}\right)^{*}}^{q}
$$

For a fixed ball $B=B\left(x_{0}, 1\right)$, we decompose $f=f \chi_{2 B}+$ $f \chi_{(2 B)^{c}}:=f_{1}+f_{2}$. Since $\mathscr{T}_{\alpha, \Omega}$ is a sublinear operator, we get

$$
\begin{aligned}
& \frac{1}{w^{q}(B)^{q \lambda / p}} \int_{B}\left|\mathscr{T}_{\alpha, \Omega} f(x)\right|^{q} w(x)^{q} d x \\
& \leq \frac{1}{w^{q}(B)^{q \lambda / p}} \int_{B}\left(\left|\mathscr{T}_{\alpha, \Omega} f_{1}(x)\right|^{q}\right. \\
& \left.\quad+\left|\mathscr{T}_{\alpha, \Omega} f_{2}(x)\right|^{q}\right) w^{q}(x) d x \\
& :=K+K K .
\end{aligned}
$$

To estimate the term $K$, using the fact that $\mathscr{T}_{\alpha, \Omega}$ is bounded from $L^{p}\left(w^{p}\right)$ to $L^{q}\left(w^{q}\right)$ with $w \in A_{\left(p / r^{\prime}, q\right)}$, we can get

$$
\begin{aligned}
& \int_{B}\left|\mathscr{T}_{\alpha, \Omega} f_{1}(x)\right|^{q} w^{q}(x) d x \\
& \quad \leq C\|f\|_{M_{p, \lambda}\left(w^{p}, w^{q}\right)}^{q} w^{q}(B)^{q \lambda / p},
\end{aligned}
$$

which implies that

$$
K \leq C\|f\|_{M_{p, \lambda}\left(w^{p}, w^{q}\right)} \cdot
$$

For the term $K K$, by the similar arguments as that of Theorem 1, we obtain

$$
\begin{aligned}
K K \leq & C \sum_{k}\left(2^{-k(n-\alpha)} \int_{A_{k}}|\Omega(x-y)||f(y)| d y\right)^{q} \\
& \times w^{q}(B)^{1-q \lambda / p} \\
\leq & C \sum_{k}\left[2^{-k(n-\alpha)+n k / r}\left(|f(y)|^{r^{\prime}} d y\right)^{1 / r^{\prime}}\right]^{q} \\
& \times w^{q}(B)^{1-q \lambda / p} \\
\leq & C\|f\|_{M_{p, \lambda}\left(w^{p}, w^{q}\right)} \\
& \times\left(\sum_{k=1}^{\infty} \frac{w^{q}(B)^{(1 / q-\lambda / p)}}{w^{q}\left(2^{k+1} B\right)^{(1 / q-\lambda / p)}}\right)^{q} \\
\leq & C\|f\|_{M_{p, \lambda}\left(w^{p}, w^{q}\right)^{\cdot}}^{q}
\end{aligned}
$$

We have completed the proof of $(a)$.

We will omit the proof of $(b)$ since we can prove it by using $A_{(1, q)}$ condition and the weak type estimates of $\mathscr{T}_{\alpha, \Omega}$ similar to the proof of Theorem 2 .

\section{Boundedness of Commutators}

We say that $b$ is a $\operatorname{BMO}\left(\mathbb{R}^{n}\right)$ function if the following sharp maximal function is finite:

$$
b^{\#}(x)=\sup _{B} \frac{1}{|B|} \int_{B}\left|b(y)-b_{B}\right| d y,
$$

where the supreme is taken over all balls $B \subset \mathbb{R}^{n}$ and $f_{B}=$ $(1 /|B|) \int_{B} f(y) d y$. This means $\|b\|_{\mathrm{BMO}\left(\mathbb{R}^{n}\right)}=\left\|b^{\#}\right\|_{L^{\infty}}<+\infty$. 
An early work about $\mathrm{BMO}\left(\mathbb{R}^{n}\right)$ space can be attributed to John and Nirenberg [22]. For $1<p<\infty$, there is a close relation between $\mathrm{BMO}\left(\mathbb{R}^{n}\right)$ and $A_{p}$ weights:

$$
\operatorname{BMO}\left(\mathbb{R}^{n}\right)=\left\{\alpha \log w: w \in A_{p}, \alpha \geq 0\right\}
$$

Given an operator $T$ acting on a generic function $f$ and a function $b$, the commutator $T_{b}$ is formally defined as

$$
T_{b} f=[b, T] f=b T(f)-T(b f) .
$$

Since $L^{\infty}\left(\mathbb{R}^{n}\right) \subsetneq \operatorname{BMO}\left(\mathbb{R}^{n}\right)$, the boundedness of $T_{b}$ is worse than $T$ (e.g., the singularity; see also [23]). Therefore, many authors want to know whether $T_{b}$ shares the similar boundedness with $T$. There are a lot of articles that deal with the topic of commutators of different operators with BMO functions on Lebesgue spaces. The first results for this commutator were obtained by Coifman et al. [24] in their study of certain factorization theorems for generalized Hardy spaces. In the present section, we will extend the boundedness of $\mathscr{T}_{\Omega}$ and $\mathscr{T}_{\alpha, \Omega}$ to $\mathscr{T}_{\Omega, b}$ and $\mathscr{T}_{\alpha, \Omega, b}$, respectively.

Theorem 6. Let $r, p, \lambda$, and $w$ be as in Theorem 1. Suppose that the sublinear operator $\mathscr{T}_{\Omega}$ satisfies condition (5) for any integral function $f$ with compact support. If $\mathscr{T}_{\Omega, b}$ is bounded on $L^{p}(w)$ with $b \in B M O\left(\mathbb{R}^{n}\right)$, then $\mathscr{T}_{\Omega, b}$ is bounded on $M_{p, \lambda}(w)$.

Theorem 7. Let $p, r, q, \alpha, w$, and $\lambda$ be as in Theorem 3(a) and let the sublinear operator $\mathscr{T}_{\alpha, \Omega}$ satisfy condition (18) for any integral function $f$ with compact support. If $\mathscr{T}_{\alpha, \Omega, b}$ maps $L^{p}\left(w^{p}\right)$ into $L^{q}\left(w^{q}\right)$ with $b \in B M O\left(\mathbb{R}^{n}\right)$, then $\mathscr{T}_{\alpha, \Omega, b}$ is bounded from $M_{p, \lambda}\left(w^{p}, w^{q}\right)$ to $M_{q, q \lambda / p}\left(w^{q}\right)$.

The following lemmas about $\operatorname{BMO}\left(\mathbb{R}^{n}\right)$ functions will help us to prove Theorems 6 and 7 .

Lemma 8 (see [25, Theorem 3.8]). Let $1 \leq p<\infty$ and $b \in$ $B M O\left(\mathbb{R}^{n}\right)$. Then for any ball $B \subset \mathbb{R}^{n}$, the following statements are true.

(a) There exist constants $C_{1}$ and $C_{2}$ such that for all $\alpha>0$

$$
\left|\left\{x \in B:\left|b(x)-b_{B}\right|>\alpha\right\}\right| \leq C_{1}|B| e^{-C_{2} \alpha /\|b\|_{B M O\left(\mathbb{R}^{n}\right)}} .
$$

(b) Inequality (49) is called John-Nirenberg inequality:

$$
\left|b_{2^{\lambda} B}-b_{B}\right| \leq 2^{n} \lambda\|b\|_{B M O\left(\mathbb{R}^{n}\right)} .
$$

Lemma 9 ([1, Proposition 7.1.2] (see also [14, Theorem 5])). Let $w \in A_{\infty}$ and $1<p<\infty$. Then the following statements are equivalent:

(a) $\|b\|_{B M O\left(\mathbb{R}^{n}\right)} \sim \sup _{B}\left((1 /|B|) \int_{B}\left|b(x)-b_{B}\right|^{p} d x\right)^{1 / p}$,

(b) $\|b\|_{B M O\left(\mathbb{R}^{n}\right)} \sim \sup _{B} \inf _{a \in \mathbb{R}}(1 /|B|) \int_{B}|b(x)-a| d x$,

(c) $\|b\|_{B M O(w)}=\sup _{B}(1 / w(B)) \int_{B}\left|b(x)-b_{B, w}\right| w(x) d x$, where $B M O(w)=\left\{b:\|b\|_{B M O(w)}<\infty\right\}$ and $b_{B, w}=$ $(1 / w(B)) \int_{B} b(y) w(y) d y$.
Lemma 10. Let $p, r, b$, and $w$ be as in Theorem 6 and let $B=$ $B\left(x_{0}, 1\right)$ be a generic fixed ball. Then the inequality

$$
\begin{gathered}
\left(\int_{\left|x_{0}-y\right|>2} \frac{\left|\Omega\left(x_{0}-y\right) f(y)\right|}{\left|x_{0}-y\right|^{n}}\left|b_{B, w}-b(y)\right| d y\right)^{p} \\
\leq C\|f\|_{M_{p, \lambda}(w)}^{p} w(B)^{\lambda-1}
\end{gathered}
$$

holds for every $y \in(2 B)^{c}$, where $(2 B)^{c}=\mathbb{R}^{n} \backslash(2 B)$.

Proof. We will consider two cases.

Case $1\left(P>r^{\prime}\right)$. In this case, $w \in A_{p / r^{\prime}}$. Using Hölder's inequality and Lemma 5 to the left-hand side of (51), we have

$$
\begin{aligned}
& \int_{\left|x_{0}-y\right|>2} \frac{\left|\Omega\left(x_{0}-y\right) f(y)\right|}{\left|x_{0}-y\right|^{n}}\left|b_{B, w}-b(y)\right| d y \\
& \leq \sum_{j=1}^{\infty} \int_{2^{j}<\left|x_{0}-y\right|<2^{j+1}} \frac{\left|\Omega\left(x_{0}-y\right) f(y)\right|}{\left|x_{0}-y\right|^{n}} \\
& \quad \times\left|b_{B, w}-b(y)\right| d y \\
& \leq \sum_{j=1}^{\infty} \frac{1}{\left|2^{j} B\right|} \int_{A_{j}}\left|\Omega\left(x_{0}-y\right) f(y)\right|\left|b_{B, w}-b(y)\right| d y \\
& \leq C\|f\|_{M_{p, \lambda}(w)} \sum_{j=1}^{\infty} \frac{2^{n j / r} w\left(2^{j+1} B\right)^{\lambda / p}}{\left|2^{j} B\right|} \\
& \quad \times\left(\int_{2^{j+1} B}\left|b_{B, w}-b(y)\right|^{p^{\prime} /\left(p-r^{\prime}\right)}\right. \\
& \left.\quad \times w(y)^{-r^{\prime} /\left(p-r^{\prime}\right)} d y\right)^{\left(p-r^{\prime}\right) / p r^{\prime}} .
\end{aligned}
$$

Set

$$
A=\left(\int_{2^{j+1} B}\left|b_{B, w}-b(y)\right|^{\bar{p}^{\prime} r^{\prime}} w^{1-\bar{p}^{\prime}} d y\right)^{1 / \bar{p}^{\prime} r^{\prime}}
$$

where $\bar{p}=p / r^{\prime}>1$. Thus

$$
\begin{aligned}
& A \leq\left(\int _ { 2 ^ { j + 1 } B } \left(\left|b_{2^{j+1} B, w^{1-\bar{p}^{\prime}}}-b(y)\right|\right.\right. \\
& \left.+\left|b_{2^{j+1} B, w^{1-\bar{p}^{\prime}}}-b_{B, w}\right|\right)^{\bar{p}^{\prime} r^{\prime}} \\
& \left.\times w(y)^{1-\bar{p}^{\prime}} d y\right)^{1 / \bar{p}^{\prime} r^{\prime}} \\
& \leq\left(\int_{2^{j+1} B}\left|b_{2^{j+1} B, w^{1-\bar{p}^{\prime}}}-b(y)\right|^{\bar{p}^{\prime} r^{\prime}}\right. \\
& \left.\times w(y)^{1-\bar{p}^{\prime}} d y\right)^{1 / \bar{p}^{\prime} r^{\prime}}
\end{aligned}
$$




$$
\begin{aligned}
& +\left|b_{2^{j+1} B, w^{1-\bar{p}^{\prime}}}-b_{B, w}\right| \\
& \times w^{1-\bar{p}^{\prime}}\left(2^{j+1} B\right)^{1 / \bar{p}^{\prime} r^{\prime}} \\
=: & A_{1}+A_{2} .
\end{aligned}
$$

Lemma 9 implies that

$$
A_{1} \leq C w^{1-\bar{p}^{\prime}}\left(2^{j+1} B\right)^{1 / \bar{p}^{\prime} r^{\prime}} .
$$

We are now in a position to deal with $A_{2}$; by (50), we have

$$
\begin{aligned}
& \left|b_{2^{j+1} B, w^{1-\bar{p}^{\prime}}}-b_{B, w}\right| \\
& \leq\left|b_{2^{j+1} B, w^{1-\bar{p} \prime}}-b_{2^{j+1} B}\right| \\
& +\left|b_{2^{j+1} B}-b_{B}\right|+\left|b_{B}-b_{B, w}\right| \\
& \leq \frac{1}{w^{1-\bar{p}^{\prime}}\left(2^{j+1} B\right)} \\
& \times \int_{2^{j+1} B}\left|b(y)-b_{2^{j+1} B}\right| w(y)^{1-\bar{p}^{\prime}} d y \\
& +2^{n}(j+1)\|b\|_{\mathrm{BMO}\left(\mathbb{R}^{n}\right)} \\
& +\frac{1}{w(B)} \int_{B}\left|b(y)-b_{B}\right| w(y) d y \\
& :=A_{21}+A_{22}+A_{23} \text {. }
\end{aligned}
$$

Combining (23) with (49), we have

$$
\begin{aligned}
A_{23}= & \frac{1}{w(B)} \\
& \times \int_{0}^{\infty} w\left(\left\{x \in B:\left|b(y)-b_{B}\right|>\alpha\right\}\right) d \alpha \\
\leq & C \int_{0}^{\infty} e^{-C_{2} \alpha \delta /\|b\|_{\text {Bмо }\left(\mathbb{R}^{n}\right)}} d \alpha \\
\leq & C .
\end{aligned}
$$

In the same manner we can see that

$$
A_{21} \leq C \text {. }
$$

It follows immediately that

$$
A_{2} \leq C\left(2^{n}(j+1)+2\right) w^{1-\bar{p}^{\prime}}\left(2^{j+1} B\right)^{1 / \bar{p}^{\prime} r^{\prime}} .
$$

Therefore

$$
A \leq C(j+1) w^{1-\bar{p}^{\prime}}\left(2^{j+1} B\right)^{1 / \bar{p}^{\prime} r^{\prime}} .
$$

A further use of (21) and $w \in p / r^{\prime}$ allow us to obtain

$$
\begin{aligned}
& \sum_{j=1}^{\infty} \frac{2^{n j / r} w\left(2^{j+1} B\right)^{\lambda / p}}{\left|2^{j} B\right|} \\
& \quad \times\left(\int_{2^{j+1} B}\left|b(y)-b_{B, w}\right|^{\bar{p}^{\prime} r^{\prime}} w(y)^{1-\bar{p}^{\prime}} d y\right)^{1 / \bar{p}^{\prime} r^{\prime}}
\end{aligned}
$$

$$
\begin{aligned}
\leq & \sum_{j=1}^{\infty} \frac{2^{n j / r} w\left(2^{j+1} B\right)^{\lambda / p}}{\left|2^{j} B\right|} \\
& \times(j+1) w(y)^{1-\vec{p}^{\prime}}\left(2^{j+1} B\right)^{1 / \bar{p}^{\prime} r^{\prime}} \\
\leq & C \sum_{j=1}^{\infty} \frac{2^{n j / r}\left|2^{j+1} B\right|^{1 / r^{\prime}}(j+1)}{\left|2^{j} B\right|} \\
& \times \frac{w(B)^{(1-\lambda) / p}}{w\left(2^{j+1} B\right)^{(1-\lambda) / p}} w(B)^{(\lambda-1) / p} \\
\leq & C \sum_{j=1}^{\infty} \frac{j+1}{D^{(j+1)(1-\lambda) / p}} w(B)^{(\lambda-1) / p} \\
\leq & C w(B)^{(\lambda-1) / p},
\end{aligned}
$$

where $D>1$ is a constant that appeared in (21).

Case $2\left(P=r^{\prime}\right)$. In this case, $w \in A_{1}$. We can prove (51) by a similar analysis as in the proof of Theorem 1 (in the case $\left.P=r^{\prime}\right)$ and Case 1 .

Having disposed of the previous preliminary step, we can now return to the proofs of Theorems 6 and 7 .

Proof of Theorem 6. The task is now to find a constant $C$ such that for fixed ball $B=B\left(x_{0}, 1\right)$, we can obtain

$$
\begin{aligned}
& \frac{1}{w(B)^{\lambda}} \int_{B}\left|\mathscr{T}_{\Omega, b} f(x)\right|^{p} w(x) d x \\
& \leq C\|f\|_{M_{p, \lambda}(w)}^{p} .
\end{aligned}
$$

We decompose $f=f \chi_{2 B}+f \chi_{(2 B)^{c}}:=f_{1}+f_{2}$ and consider the corresponding splitting

$$
\begin{aligned}
& \int_{B}\left|\mathscr{T}_{\Omega, b} f(x)\right|^{p} w(x) d x \\
& \leq C\left(\int_{B}\left|\mathscr{T}_{\Omega, b} f_{1}(x)\right|^{p} w(x) d x\right. \\
& \left.\quad \quad+\int_{B}\left|\mathscr{T}_{\Omega, b} f_{2}(x)\right|^{p} w(x) d x\right) \\
& =: L+L L .
\end{aligned}
$$

It follows from the $L^{p}(w)$ boundedness of $\mathscr{T}_{\Omega, b}$ and $w \in$ $A_{p / r^{\prime}} \subset A_{p}$ that

$$
\begin{aligned}
L & \leq C \int_{2 B}|f(x)|^{p} w(x) d x \\
& \leq C\|f\|_{M_{p, \lambda}(w)}^{p} w(B)^{\lambda} .
\end{aligned}
$$


Then a further use of (5) derives that

$$
\begin{aligned}
& \left|\mathscr{T}_{\Omega, b} f_{2}(x)\right|^{p} \\
& \leq C\left(\int_{\mathbb{R}^{n}} \frac{\left|\Omega(x-y) f_{2}(y)\right||b(x)-b(y)|}{|x-y|^{n}} d y\right)^{p} \\
& \leq C\left(\int_{\left|x_{0}-y\right|>2} \frac{\left|\Omega\left(x_{0}-y\right) f(y)\right|}{\left|x_{0}-y\right|^{n}}\right. \\
& \left.\quad \times\left\{\left|b(x)-b_{B, w}\right|+\left|b_{B, w}-b(y)\right|\right\} d y\right)^{p} .
\end{aligned}
$$

Therefore

$$
\begin{array}{r}
L L \leq C\left(\int_{\left|x_{0}-y\right|>2} \frac{\left|\Omega\left(x_{0}-y\right) f(y)\right|}{\left|x_{0}-y\right|^{n}} d y\right)^{p} \\
\quad \times \int_{B}\left|b(x)-b_{B, w}\right|^{p} w(x) d x \\
+C\left(\int_{\left|x_{0}-y\right|>2} \frac{\left|\Omega\left(x_{0}-y\right) f(y)\right|}{\left|x_{0}-y\right|^{n}}\right. \\
\left.\quad \times\left|b(y)-b_{B, w}\right| d y\right)^{p} w(B): \\
=L L_{1}+L L_{2} .
\end{array}
$$

By Lemma 10, we have

$$
L L_{2} \leq C\|f\|_{M_{p, \lambda}(w)}^{p} w(B)^{\lambda}
$$

We proceed to estimate $L L_{1}$. Without loss of generality, we assume that $p>r^{\prime}$. Taking into account (20), (22), and Lemma 9, we have

$$
\begin{aligned}
L L_{1}= & \left(\sum_{j=1}^{\infty} \int_{2^{j}<\left|x_{0}-y\right|<2^{j+1}} \frac{\left|\Omega\left(x_{0}-y\right) f(y)\right|}{\left|x_{0}-y\right|^{n}} d y\right)^{p} \\
& \times \int_{B}\left|b(x)-b_{B, w}\right|^{p} w(x) d x \\
\leq & \left(\sum_{j=1}^{\infty} \frac{1}{2^{j} B \mid} \int_{A_{j}}\left|\Omega\left(x_{0}-y\right)\right|^{r} d y\right)^{1 / r} \\
& \times\left(\int_{2^{j+1} B}|f(y)|^{r^{\prime}} d y\right)^{1 / r^{\prime}} \\
& \times \int_{B}\left|b(x)-b_{B, w}\right|^{p} w(x) d x
\end{aligned}
$$$$
\leq C\left[\sum_{j=1}^{\infty} \frac{2^{n j / r}}{\left|2^{j} B\right|}\right.
$$$$
\times\left(\int_{2^{j+1} B}|f(y)|^{p} w(y) d y\right)^{1 / p}
$$$$
\left.\times\left(\int_{2^{j+1} B} w(y)^{-r^{\prime} /\left(p-r^{\prime}\right)} d y\right)^{\left(p-r^{\prime}\right) / p r^{\prime}}\right]^{p}
$$$$
\times \int_{B}\left|b(x)-b_{B, w}\right|^{p} w(x) d x
$$$$
\leq C\|f\|_{M_{p, \lambda}(w)}^{p}
$$$$
\times\left(\sum_{j=1}^{\infty} \frac{w(B)^{(1-\lambda) / p}}{w\left(2^{j+1} B\right)^{(1-\lambda) / p}}\right)^{p} w(B)^{\lambda}
$$$$
\leq C\|f\|_{M_{p, \lambda}(w)}^{p} w(B)^{\lambda} \text {. }
$$

Hence

$$
L L \leq C\|f\|_{M_{p, \lambda}(w)}^{p} w(B)^{\lambda} .
$$

According to (64) and (69), we have completed the proof of Theorem 6.

Proof of Theorem 7. The proof of Theorem 7 is similar to that of Theorem 6, except using $w \in A_{(p, q)}$. We omit its proof here.

\section{Acknowledgments}

The authors thank Professor Shanzhen Lu and the referee for their valuable suggestions. This work was partially supported by NSF of China (Grants nos. 11271175, 10901076, and 11171345) and NSF of Shandong Province (Grant no. ZR2012AQ026).

\section{References}

[1] L. Grafakos, Classical and Modern Fourier Analysis, Pearson Education, 2004.

[2] J. Duoandikoetxea, "Weighted norm inequalities for homogeneous singular integrals," Transactions of the American Mathematical Society, vol. 336, no. 2, pp. 869-880, 1993.

[3] Y. Ding, D. S. Fan, and Y. B. Pan, "Weighted boundedness for a class of rough Marcinkiewicz integrals," Indiana University Mathematics Journal, vol. 48, no. 3, pp. 1037-1055, 1999.

[4] Y. Ding, S. Z. Lu, and K. Yabuta, "On commutators of Marcinkiewicz integrals with rough kernel," Journal of Mathematical Analysis and Applications, vol. 275, no. 1, pp. 60-68, 2002.

[5] D. S. Fan and Y. B. Pan, "Singular integral operators with rough kernels supported by subvarieties," American Journal of Mathematics, vol. 119, no. 4, pp. 799-839, 1997.

[6] S. Z. Lu, D. C. Yang, and G. E. Hu, Herz Type Spaces and their Applications, Science Press, Beijing, China, 2008. 
[7] A. Seeger, "Singular integral operators with rough convolution kernels," Journal of the American Mathematical Society, vol. 9, no. 1, pp. 95-105, 1996.

[8] P. Sjögren and F. Soria, "Rough maximal functions and rough singular integral operators applied to integrable radial functions," Revista Matemática Iberoamericana, vol. 13, no. 1, pp. 118, 1997.

[9] G. E. Hu, S. Z. Lu, and D. C. Yang, "Boundedness of rough singular integral operators on homogeneous Herz spaces," Journal of the Australian Mathematical Society, vol. 66, no. 2, pp. 201-223, 1999.

[10] F. Soria and G. Weiss, "A remark on singular integrals and power weights," Indiana University Mathematics Journal, vol. 43, no. 1, pp. 187-204, 1994.

[11] D. K. Watson and R. L. Wheeden, "Norm estimates and representations for Calderón-Zygmund operators using averages over starlike sets," Transactions of the American Mathematical Society, vol. 351, no. 10, pp. 4127-4171, 1999.

[12] H. Ojanen, Weighted norm inequalities for rough singular integrals [Doctoral Dissertation], The State University of New Jersey, New Jersey, NJ, USA, 1999.

[13] X. W. Li and D. C. Yang, "Boundedness of some sublinear operators on Herz spaces," Illinois Journal of Mathematics, vol. 40, no. 3, pp. 484-501, 1996.

[14] B. Muckenhoupt and R. L. Wheeden, "Weighted bounded mean oscillation and the Hilbert transform," Studia Mathematica, vol. 54, no. 3, pp. 221-237, 1976.

[15] Y. Komori and S. Shirai, "Weighted Morrey spaces and a singular integral operator," Mathematische Nachrichten, vol. 282, no. 2, pp. 219-231, 2009.

[16] B. Muckenhoupt and R. L. Wheeden, "Weighted norm inequalities for singular and fractional integrals," Transactions of the American Mathematical Society, vol. 161, pp. 249-258, 1971.

[17] S. Chanillo, D. K. Watson, and R. L. Wheeden, "Some integral and maximal operators related to starlike sets," Studia Mathematica, vol. 107, no. 3, pp. 223-255, 1993.

[18] Y. Ding and S. Z. Lu, "Weighted norm inequalities for fractional integral operators with rough kernel," Canadian Journal of Mathematics, vol. 50, no. 1, pp. 29-39, 1998.

[19] Y. Ding and S. Z. Lu, "Homogeneous fractional integrals on Hardy spaces," The Tohoku Mathematical Journal, vol. 52, no. 1, pp. 153-162, 2000.

[20] D. S. Fan, S. Z. Lu, and D. C. Yang, "Regularity in Morrey spaces of strong solutions to nondivergence elliptic equations with VMO coefficients," Georgian Mathematical Journal, vol. 5, no. 5, pp. 425-440, 1998.

[21] S. Z. Lu and Y. Zhang, "Criterion on $L^{p}$-boundedness for a class of oscillatory singular integrals with rough kernels," Revista Matemática Iberoamericana, vol. 8, no. 2, pp. 201-219, 1992.

[22] F. John and L. Nirenberg, "On functions of bounded mean oscillation," Communications on Pure and Applied Mathematics, vol. 14, pp. 415-426, 1961.

[23] C. Pérez, "Endpoint estimates for commutators of singular integral operators," Journal of Functional Analysis, vol. 128, no. 1, pp. 163-185, 1995.

[24] R. R. Coifman, R. Rochberg, and G. Weiss, "Factorization theorems for Hardy spaces in several variables," Annals of Mathematics, vol. 103, no. 3, pp. 611-635, 1976.

[25] J. García-Cuerva and J. L. Rubio de Francia, Weighted Norm Inequalities and Related Topics, North-Holland Publishing, Amsterdam, The Netherlands, 1985. 


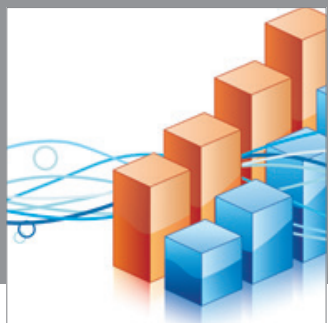

Advances in

Operations Research

mansans

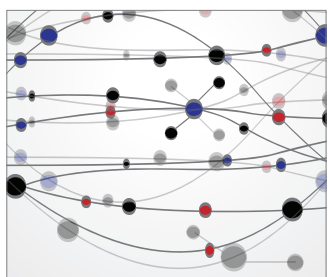

The Scientific World Journal
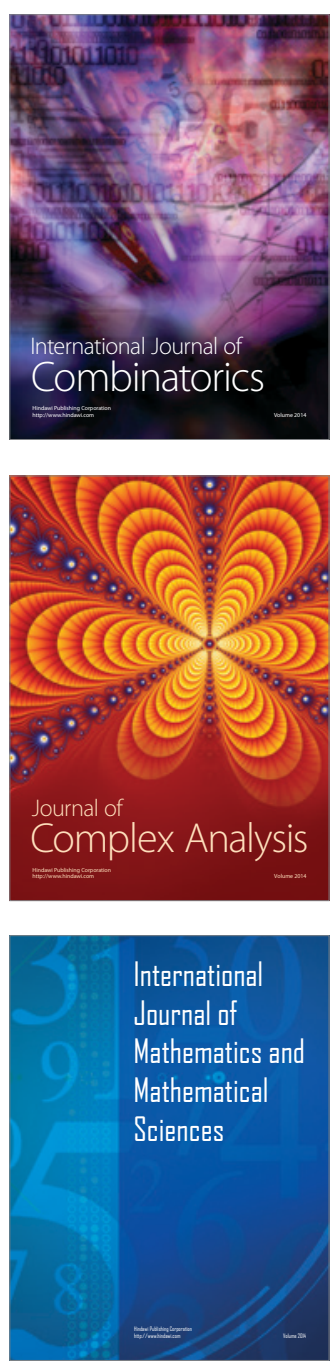
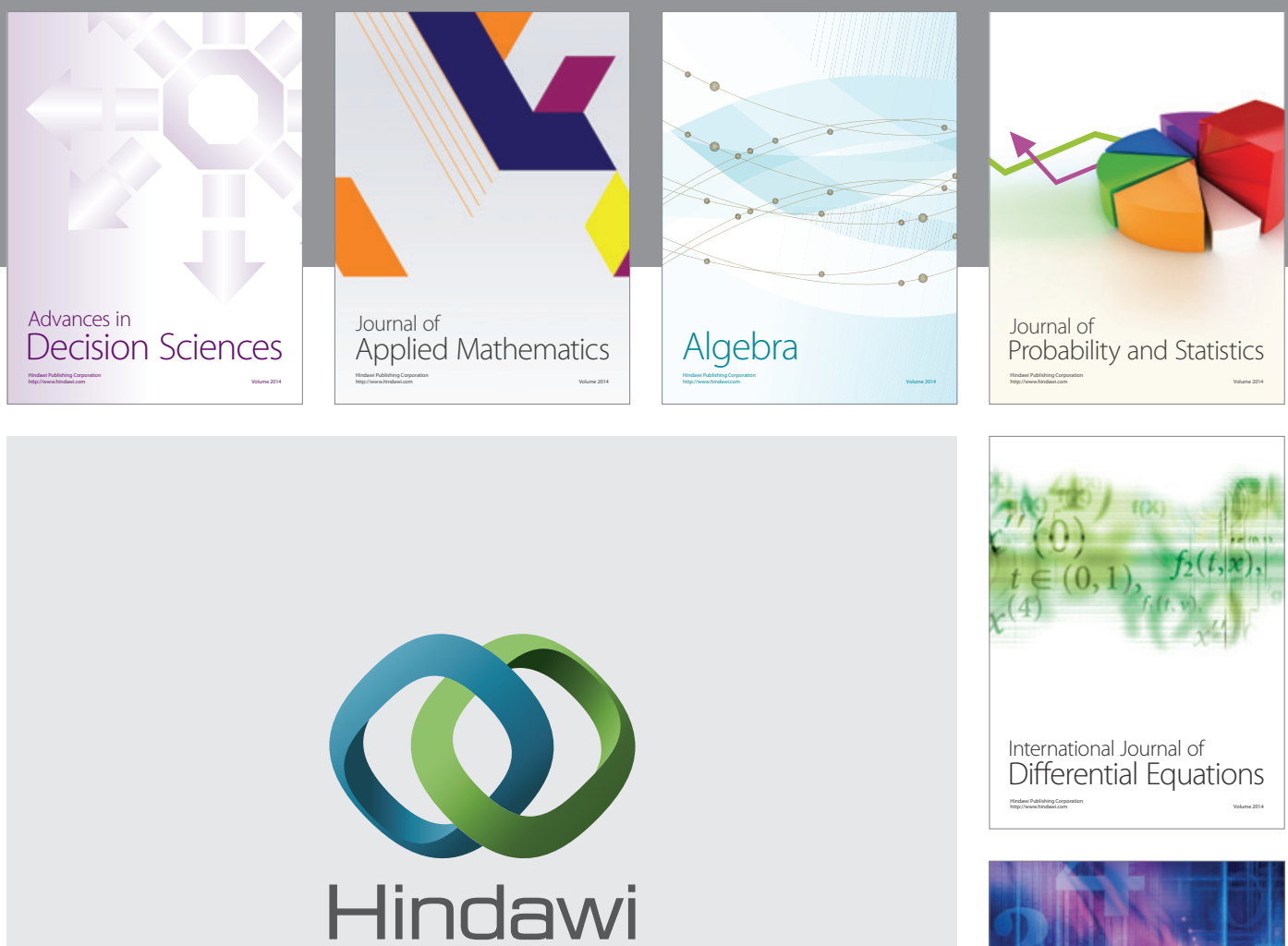

Submit your manuscripts at http://www.hindawi.com
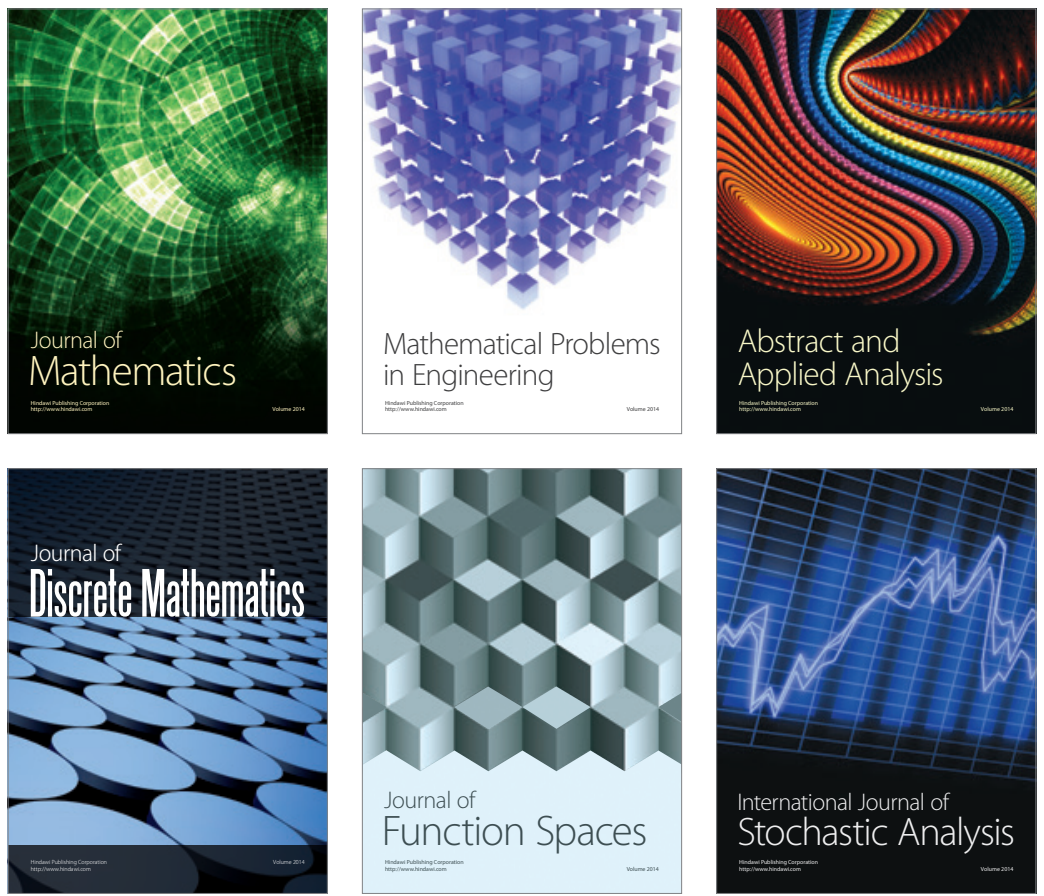

Journal of

Function Spaces

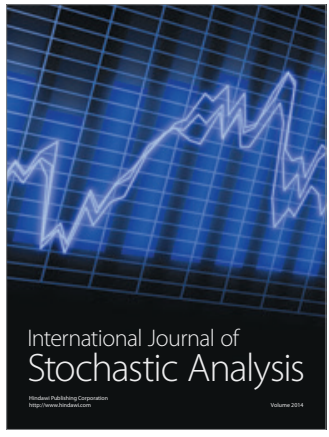

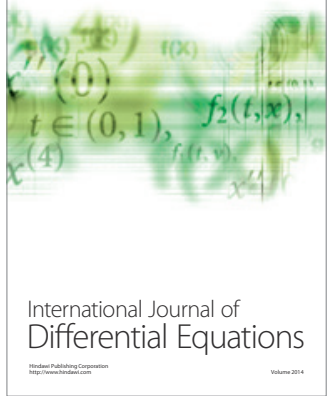
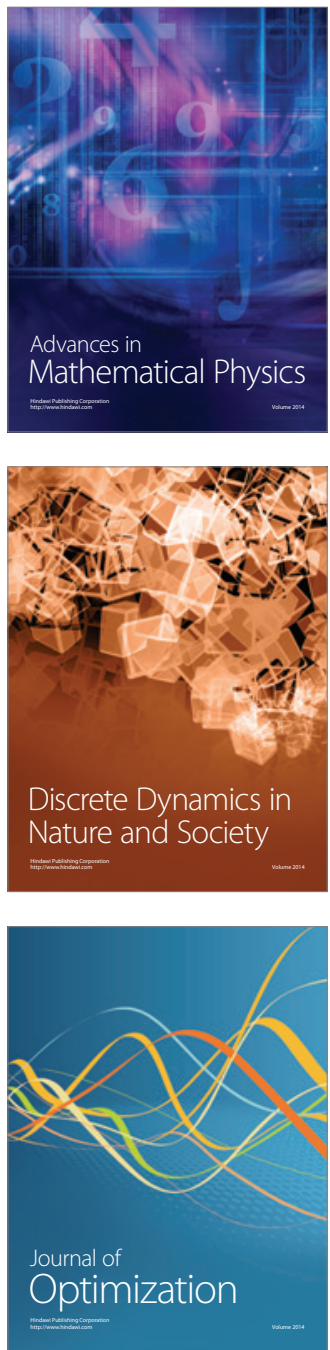